Verhaltenstherapie 1991;1:189-190

\title{
Contents, Vol. 1, No. 3, 1991
}

Verhaltenstherapie

Band 1, Heft 3, September 1991

Inhalt

Editorial Die Verhaltenstherapie und das «Forschungsgutachten zu Fragen eines

Psychotherapeutengesetzes»

Hand, 191

Originalarbeiten Formale und inhaltliche Aspekte depressiver Kognitionen

Linden, M., Simons, G 200

Reaktionen auf depressive und nichtdepressive Selbstdar-stellungen depressiver und

nichtdepressiver Patientinnen

Hautzinger, M., Heckel-Guhrenz, S 207

Streßbewältigungsstrategien schizophrener Patienten: Eine Pilotstudie

Kraemer, S., Schickor, 1212

Ambulante psychotherapeutische Vorbehandlungen bei Patienten einer medizinisch-

psychosomatischen Klinik

Rief, W, Leibl, C, Fichter, M.M 219

Mitteilungen der Verbände

Arbeitsgemeinschaft für Verhaltensmodifikation

(AVM-Ö/D) 240

Bayerische Private Akademie für Psychotherapie (BAP)

Centrum für Integrative Psychotherapie (CIP)

Deutsche Akademie für Verhaltenstherapie (DAVT)

Deutsche Gesellschaft für Verhaltenstherapie (DGVT)

Fachverband klinische Verhaltenstherapie e.V. (FKV) 250

Österreichische Gesellschaft für Verhaltenstherapie (ÖGVT) . . 252

Vereinigung der Kassenpsychologen

253

Ausbildungsinstitut für Klinische Verhaltenstherapie in NRW

(AFKV in NRW e.V.) 256

Verein zur Förderung der klinischen Verhaltenstherapie

(VFKV)

257

Tagungen und Kongresse 259

Impressum 189

Inhaltsverzeichnis 190

Hinweise für Autoren 263

Aus der Praxis für die Praxis

Die Yale-Brown Obsessive Compulsive Scale (Y-BOCS): Ein halbstrukturiertes Interview zur Beurteilung des Schweregrades von Denk- und Handlungszwängen

Hand, I., Büttner-Westphal, H 
Neuigkeiten vom Buch- und Zeitschriftenmarkt Bücher über bulimische und anorektische Eßstörungen: Eine kommentierende Bibliographie

Fichter, M. M 234 